\title{
Penetrating Ureteral Trauma
}

\section{Gustavo P. Fraga, Gustavo M. Borges, Mario Mantovani, Ubirajara Ferreira, Tiago L. Laurito, Nelson R. Netto Jr}

Division of Trauma Surgery, School of Medicine, State University of Campinas, Unicamp, Campinas, Sao Paulo, Brazil

\begin{abstract}
Objective: The purpose of this series is to report our experience in managing ureteral trauma, focusing on the importance of early diagnosis, correct treatment, and the impact of associated injuries on the management and morbid-mortality.

Materials and Methods: From January 1994 to December 2002, 1487 laparotomies for abdominal trauma were performed and 20 patients with ureteral lesions were identified, all of them secondary to penetrating injury. Medical charts were analyzed as well as information about trauma mechanisms, diagnostic routine, treatment and outcome.

Results: All patients were men. Mean age was 27 years. The mechanisms of injury were gunshot wounds in 18 cases (90\%) and stab wounds in two (10\%). All penetrating abdominal injuries had primary indication of laparotomy, and neither excretory urography nor computed tomography were used in any case before surgery. The diagnosis of ureteric injury was made intra-operatively in 17 cases $(85 \%)$. Two ureteral injuries $(10 \%)$ were initially missed. All patients had associated injuries. The treatment was dictated by the location, extension and time necessary to identify the injury. The overall incidence of complications was 55\%. The presence of shock on admission, delayed diagnosis, Abdominal Trauma Index > 25 , Injury Severity Score $>25$ and colon injuries were associated to a high complication rate, however, there was no statistically significant difference. There were no mortalities in this group.

Conclusions: A high index of suspicion is required for diagnosis of ureteral injuries. A thorough exploration of all retroperitoneal hematoma after penetrating trauma should be an accurate method of diagnosis; even though it failed in $10 \%$ of our cases.
\end{abstract}

Key words: ureter; wounds and injuries; reconstructive surgical procedures

Int Braz J Urol. 2007; 33: 142-50

\section{INTRODUCTION}

Ureteral lesions occur as a consequence of external trauma, open surgical procedures, laparoscopy or ureteroscopic procedures. Lesions caused by external trauma are rare. Iatrogenic damage is the most frequent cause of ureteral injury. In one review of 13 series, hysterectomy was responsible for the majority (54\%), followed by colorectal surgery (14\%), pelvic surgery (8\%) and abdominal vascular surgery (6\%) (1). Ureteral injury occurs only in $2 \%$ to $5 \%$ of the victims of abdominal gunshot wounds (2-6).

Ureteral injury is usually silent, producing no early signs or symptoms. Hematuria is typically absent on presentation, as described in several series, and urinalysis is normal at hospital admission in 15 to $55 \%$ of patients with ureteral injury $(3,4,6)$. 
Visceral injuries are commonly associated to ureteral injury and are easily identified in most cases. Patients with hemodynamic instability and extensive blood loss are more susceptible to have ureteral lesions not identified at surgical exploration $(3,7,8)$.

Once diagnosed, ureteral lesion must be primarily treated. Termino-terminal anastomosis (ureteroureterostomy), debridment and direct repair of minor lesions and ureteral reimplantation are treatment options for almost all ureteral lesions $(3,9,10)$. Transureteroureterostomy, ileum interposition and auto-transplantation are performed in extreme cases of extended ureteral loss. Patients with shock at admission, massive intraoperative blood loss, associated lesions and local contamination have high morbidity rates and primary repair of ureteral injury in these cases have been questioned.

The purpose of this series is to report our experience in ureteral trauma management, with attention to the diagnosis, repair, and outcome of these injuries.

\section{MATERIALS AND METHODS}

The trauma surgery division is responsible for traumatic and non-traumatic surgical emergencies in a metropolitan region consisting of 2.4 million people. From January 1994 to December 2002, 1487 laparotomies for abdominal trauma were performed. Penetrating trauma was responsible for $69.1 \%$ of the operation and blunt trauma for $30.9 \%$. The sequence of diagnostic and therapeutic procedures adopted was in agreement with standards established by the Advanced Life Trauma Support (ATLS). Indications for laparotomy in patients sustained penetrating trauma were based in mechanism of trauma, physical examination, and the patient's response to initial fluid resuscitation. All patients with gunshot wounds penetrating the peritoneal cavity or retroperitoneum, and all patients with stab wounds penetrating the anterior peritoneum with hypotension, peritonitis, or evisceration underwent laparotomy, without any further investigation including computed tomography (CT) scan or intravenous urography (IVU).
In this period, 20 patients with ureteral lesions were identified, all of them secondary to penetrating injury. Surgical repair of these lesions was performed in consultation with the division of urology of our institution.

Data of all patients were analyzed in Epi-Info 6.04 computer program. In all cases, gender, age, mechanism of injury, investigation, site and grade of lesion, repair, complications and follow-up were analyzed. Physiologic condition was evaluated by Revised Trauma Score (RTS) (11), associated lesions by Abdominal Trauma Index (ATI) (12) and Injury Severity Score (ISS) (13), and mortality by TRISS (14). Ureteral lesions were classified according to the Organ Injury Scaling (OIS) (15) classification described in Table-1.

All repairs included adequate debridement of ureteral margins, spatulation, suture using a 4 or 5 zero absorbable sutures, drain, bladder catheter drainage and antibiotic prophylaxis.

The data were analyzed using nonparametric statistical methods. The principal test used was the chi-squared using the Yates correction for 2 by 2 contingency tables. A significance level of $p<0.05$ was used for all tests.

\section{RESULTS}

Ureteral lesions were identified in $1.3 \%$ considering all laparotomies and $1.9 \%$ in laparotomies for penetrating trauma.

All 20 patients included in this report were men. Age ranged from 17 to 48 years, with an aver-

Table 1 - Classification of ureteral lesions, based on Moore et al. (15).

\begin{tabular}{ll} 
Grade & Description of Injury \\
\hline I & $\begin{array}{l}\text { Contusion or hematoma } \\
\text { II }\end{array}$ \\
III & $>50 \%$ transection \\
IV & $\begin{array}{c}\text { Complete transection } \\
\text { devascularization }\end{array}$ \\
V & Avulsion with $>2 \mathrm{~cm}$ devascularization \\
\hline
\end{tabular}


age of 27 years. The mechanisms of injury were gunshot wounds in 18 cases $(90 \%)$ and stab wounds in two $(10 \%)$.

At admission, 15 patients (75\%) were hemodynamically stable and five patients $(25 \%)$ had systolic blood pressure less than $90 \mathrm{mmHg}$. The mean Revised Trauma Score (RTS) was 7.54 (range 5.22 to 7.84). Gross hematuria was observed in two patients (associated with a renal and a bladder injury, respectively) and urinalysis was performed at admission only in six of the 20 patients, with microscopic hematuria in one case $(16.7 \%)$.

Since the patients had a clear indication for surgery, no IVU or CT scan was done preoperatively. The diagnosis of ureteric injury was made intraoperatively in 18 cases (90\%). Two ureteral injuries (10\%) were initially missed, with late diagnosis made at days 8 and 12 after the first surgical procedure. One patient had urinary leakage by abdominal drain and another presented with urinoma. Retrograde pyelogram was performed in these cases, both of them showing contrast extravasation from ureter.

All patients had other associated injuries (Table-2), with a mean Abdominal Trauma Index (ATI) of 25 (range 10-64). The Injury Severity Score (ISS) ranged from 9 to 29, with an average of 14 .

The left ureter was involved in 13 patients $(65 \%)$ and the right in 7 (35\%). There were no bilateral lesions.

Three ureteric injuries (15\%) were proximal $1 / 3,10(50 \%)$ to middle $1 / 3$ and $7(35 \%)$ to the distal $1 / 3$. Two patients had only contusion to the ureter (grade I lesion), secondary to the blast effect of high velocity missile passing in close proximity to the ureteric wall. There were 9 partial lesions (grade II injury in 5 and grade III in 4 patients) and 9 cases complete transections (grade IV injury in 2 and grade $\mathrm{V}$ in 7 patients).

The treatment was determined by the location, extent and time of diagnosis (Table-3). No intervention were done for patients with grade I lesions and one of them developed a urinary fistula at the 6th postoperative day. Since no disruption of the ureter was identified at the time of laparotomy, the ureter wall necrosed at the site of blast effect developing urinary fistula.
All 9 patients with middle 1/3 injuries were repaired by ureteroureterostomy with double $\mathrm{J}$ stent. Of the 4 patients with distal 1/3 injury diagnosed during the operation, one underwent stented (double $\mathrm{J}$ ) ureteroureterostomy and 3 ureteroneocystotomy (psoas hitch in one case and Politano-Leadbetter reimplantation in 2 cases - all stented with a $8 \mathrm{~F}$ polyvinyl feeding tube for 8 weeks). The 3 proximal lesions were all identified during surgery. Two were treated with ureteroureterostomy and stent, and one caused by stab wound was repaired primarily and stented for 8 weeks.

The two patients with missed ureteral injuries underwent endoscopic treatment with double $\mathbf{J}$

Table 2 - Associated abdominal injuries.

\begin{tabular}{lc}
\hline Organ & Number of Patients (\%) \\
\hline Small bowel & $15(75 \%)$ \\
Colon & $8(40 \%)$ \\
Inferior vena cava & $4(20 \%)$ \\
Gallbladder & $3(15 \%)$ \\
Iliac vessels & $3(15 \%)$ \\
Stomach & $3(15 \%)$ \\
Liver & $2(10 \%)$ \\
Kidney & $2(10 \%)$ \\
Duodenum & $2(10 \%)$ \\
Spleen & $1(5 \%)$ \\
Bladder & $1(5 \%)$ \\
Rectum & $1(5 \%)$ \\
Diaphragm & $1(5 \%)$ \\
\hline
\end{tabular}

Table 3 - Type of surgical repair.

\begin{tabular}{lc}
\hline Type of Surgical Repair & $\begin{array}{c}\text { Number } \\
\text { of Patients (\%) }\end{array}$ \\
\hline Ureteroureterostomy + DJ stent & $12(60 \%)$ \\
Ureteral reimplant & $3(15 \%)$ \\
Double-J stent * & $3(15 \%)$ \\
Primary suture + DJ stent & $1(5 \%)$ \\
Conservative (grade I lesion) & $1(5 \%)$ \\
\hline * Two missed injuries and one patient with grade I injury (blast \\
effect).
\end{tabular}


stent. The patient with delayed grade I injury and urinary fistula was treated with endoscopic DJ stent. Stents remained in place for a mean of 43 days (ranged from 29 to 90 ).

The overall incidence of complications was 55\% (11 cases). There were 2 cases of persistent urinary fistula. One patient had suffered multiple injuries (ATI: 40), including inferior vena cava, and sustained prolonged hypotension. He was treated with ureteroureterostomy and DJ stent, and developed a urinary fistula treated conservatively with sustained ureteral catheterization. The patient was discharged from hospital on day 19 and the catheter was removed in 90 days. The second, treated with ureteral reimplantation, was diagnosed with colorectal and small bowel injuries (ATI: 31), and moderate peritoneal contamination. He developed a ureteric-colonic fistula, with extravasation of urine by colostomy. He was treated with ureteral stenting and the fistula closed with two weeks. The patient was discharged from hospital on the 14th postoperative day, but the DJ stent was removed only with 87 days.

Other complications related to the genitourinary tract included two cases of urinary tract infection and a case of persistent hematuria (until 8th postoperative day). Complications unrelated to urinary repair included pneumonia in 2 cases, neurological sequelae in 2 cases and a wound abscess in one patient.

In this study, a delay in diagnosis was a contributory factor in morbidity related to ureteral injury, because two patients with missed lesions had prolonged hospitalization (34 and 20 days, respectively), and the first had pneumonia.

Table 4-Relationship between different factors and outcome of patients.

\begin{tabular}{|c|c|c|c|}
\hline Factors & Number of Cases & Complications & p Value \\
\hline \multicolumn{4}{|c|}{ Systolic blood pressure } \\
\hline$>90 \mathrm{mmHg}$ & 15 & $46.7 \%$ & $0.436(\mathrm{NS})$ \\
\hline$<90 \mathrm{mmHg}$ & 5 & $80 \%$ & \\
\hline \multicolumn{4}{|c|}{ Time of diagnosis } \\
\hline immediate & 18 & $50 \%$ & $0.584(\mathrm{NS})$ \\
\hline delayed & 2 & $100 \%$ & \\
\hline \multicolumn{4}{|l|}{ ATI } \\
\hline$\leq 25$ & 10 & $40 \%$ & $0.368(\mathrm{NS})$ \\
\hline$>25$ & 10 & $70 \%$ & \\
\hline \multicolumn{4}{|l|}{ ISS } \\
\hline$\leq 25$ & 18 & $50 \%$ & $0.584(\mathrm{NS})$ \\
\hline$>25$ & 2 & $100 \%$ & \\
\hline \multicolumn{4}{|l|}{ Colon injury } \\
\hline presence & 8 & $62.5 \%$ & $0.361(\mathrm{NS})$ \\
\hline absence & 12 & $50 \%$ & \\
\hline \multicolumn{4}{|c|}{ Grade of ureteral injury } \\
\hline I - III & 11 & $54.5 \%$ & $0.684(\mathrm{NS})$ \\
\hline IV or $\mathrm{V}$ & 9 & $55.6 \%$ & \\
\hline
\end{tabular}

NS = no statistically significant; ATI = Abdominal Trauma Index; ISS = Injury Severity Score. 
The presence of shock on admission, delayed diagnosis, ATI > 25, ISS > 25 and colon injuries were associated with a high complication rate, however there was no statistically significant difference (Table4). There were no deaths in this series and the mean TRISS was 0.98 . Hospitalization period ranged from 5 to 35 days, with a mean of 12.4 days.

The follow-up ranged from 1 to 15 months (mean of 5 months). Only four patients were available for one-year follow-up and they were well with no evidence of stenosis on imaging studies (IVU) performed later.

\section{COMMENTS}

The ureters are relatively well protected by surrounding structures and their small size and mobility contributes to their infrequent injury. Ureteral lesions involve less than $1 \%$ of all trauma to the genitourinary tract and are predominantly associated with penetrating injury $(3,6,10)$. Ureteral avulsion caused by blunt trauma is rare and typically occurs at the level of ureteropelvic junction in children (16). Injuries caused by blunt trauma will be recognized only by a high degree of suspicion of urologic injury, and intravenous urography (IVU) is performed in suspected cases (9). In our series, all patients were victims of penetrating trauma.

In our review, $85.3 \%$ of the patients had no evidence of blood on urinalysis. Regardless of urinalysis result, a suspected ureteral lesion must be evaluated before and during laparotomy.

There are controversies on the usefulness of preoperative or intraoperative IVU for evaluating ureteral injury secondary to penetrating trauma. Some authors observed that IVU had more than $30 \%$ of false-negative rate, and a high dose of contrast on rapid infusion IVU increases the sensitivity of this test (17). Presti et al. (3), Campbell et al. (2) and Azimuddin et al. (18) found IVU diagnostic with definite evidence of ureteric injury in $14 \%$ to $33 \%$ of cases. In our series, IVU or CT were not performed preoperatively in any patient, but they are an important tool for recognizing missed injuries in complicated cases. Retrograde pyelography is probably the most sensitive ra- diographic tool for ureteral injury diagnosis. It was done in two patients with missed injury and in another with fistula after blast injury of ureter, and demonstrated contrast extravasation in all of them.

Traumatic injury to the ureter is often undiagnosed at the time of presentation and may have been overlooked in the past, due to many reasons, including the magnitude of associated injuries and low index of suspicion. All penetrating abdominal injuries should be explored when first recognized $(2,9,10)$. Direct inspection remains the fastest and most reliable method for detecting ureteric injury. An extended exploration of the retroperitoneum is mandatory in all cases of penetrating injury to this region. In cases of gunshot wounds, especially high velocity, a meticulous exploration of the area of retroperitoneal violation must be done, to avoid missing injuries secondary to the blast effect of missiles (18). Even gross inspection may sometimes miss a blast effect and there may be a role of postoperative IVU in these cases of high velocity gunshot wounds $(2,18)$. Intravenous administration of either methylene blue or diuretics may identify the injury site when it is not obvious intra-operatively.

In our study, $10 \%$ of the patients had a delay in diagnosis. A thorough exploration of retroperitoneum was not done in these two cases. Other studies had shown a delay in diagnosis ranging from $0 \%$ to $57 \%$ $(2-6,17)$. The importance of timely recognition was demonstrated in many reports. Immediate recognition of ureteric injury was associated to better results and outcome than delayed recognition $(2,3,8,19)$. Campbell et al. (3) observed that the complication rate for patients with a delay in diagnosis was $40 \%(2 / 5)$, compared to $10 \%(1 / 10)$ when the diagnosis was made at the time of presentation. It is important to be aware for signs of potential missed injury in the postoperative period. The most important signs of urinary leakage are prolonged ileus, low-grade fever, flank tenderness and persistent drainage from operative sites (4). Endourologic management of these cases is recommended by some authors $(8,20)$. Endourologic procedures are safe and simple techniques, and will obviate the need for kidney drainage or open surgery. In our series, the two patients with late diagnosis were treated endoscopically with double J stent, with satisfactory evolution, needing no further intervention. 
However, both patients had their hospital stay extended because of the delayed diagnosis.

In this series, two patients had contusion of the ureteric wall. One patient was observed expectantly, with good evolution, and the other developed a urinary fistula. Azimuddin et al. (18) described 3 patients with contusion of the ureteric wall treated without resection. A DJ stent was used in one, while the other two were observed expectantly and recovered without complication (18). According to other authors, simple stenting contused ureter (blast effect) may be adequate treatment $(2,10)$.

Penetrating injuries of abdominal cavity rarely involve the ureter alone $(2,3,5,7,9,17,18,21)$. Associated injuries to the gastrointestinal tract are commonly present, and may modify the management of ureteral injury at initial procedure. In our study, all patients had associated injuries and a mean ATI of 25 . Presti et al. (3) observed a mean number of organs injured per patient of 3,7, a mean ISS of 20.5 and ATI of 24. Hemodynamic instability or extensive damage to intra-abdominal organs might preclude definitive repair initially. In this series, the patients with shock on admission had more complications, including a case of urinary fistula. In other studies, the presence of shock, intraoperative bleeding and multiple organ involvement, were associated with a higher morbidity and mortality in patients with ureteral injuries (7).

We attempted to classify ureteral injuries according to the Organ Injury Scaling of the American Association for the Surgery of Trauma, and as it was observed by other authors, no statistically significant correlation was found between the grade of ureteral injury and morbidity. Best et al. (21) observed that mortality increased with AAST-OIS injury grade but it was not related to the ureteral injury.

Velmahos et al. (7) identified the presence of shock on admission, intra-operative bleeding, multiple intra-abdominal organ involvement, and especially severe colonic injury requiring colectomy as predictive of a poor outcome. In our series the presence of shock on admission, delayed diagnosis, ATI > 25, ISS $>25$ and colon injuries were associated to a high complication rate, however, there was no statistically significant difference.
The accepted surgical management of ureteral injuries included adequate debridement of devitalized tissue, a water-tight, tension-free spatulated anastomosis, isolation from associated contaminated injuries, adequate drainage and ureteral stenting (2$4,8,9,17,18,21)$.

In stable patient, the preferred option for repair of proximal and mid ureteric injuries is debridement and primary ureteroureterostomy. Some authors believe that repair of all proximal injuries should include a nephrostomy tube, and others do not find this to be necessary $(2-4,9,10,17)$. We do not believe that nephrostomy diversion is necessary in cases of ureteral injury, and this procedure was not used in our series. We routinely repair ureters over an indwelling stent, removed cystoscopically after 6 weeks. We prefer to use internal ureteral stents, with double $\mathbf{J}$ stent in cases of ureteroureterostomy and feeding tube in cases of ureteroneocystostomy.

This study has limitations related to its retrospective design and small number of cases. The results of this study suggest that a large, multi-center, well-designed prospective study is needed to evaluate and compare diagnostic approaches for ureteric injuries and to establish an effective treatment algorithm.

\section{CONCLUSIONS}

A high index of suspicion is required to enable surgeons to make the diagnosis of ureteral injury as promptly as possible, however, in $10 \%$ of our cases the injury was initially missed. The majority of cases $(80 \%)$ were treated successfully by primary repair.

The overall incidence of complications was high in patients with shock on admission, delayed diagnosis, multiple intra-abdominal organ involvement (ATI and ISS higher than 25), and colonic injury, but no statistically significant correlation was found between different factors and morbidity.

\section{CONFLICT OF INTEREST}

None declared. 


\section{REFERENCES}

1. St Lezin MA, Stoller ML: Surgical ureteral injuries. Urology. 1991; 38: 497-506.

2. Campbell EW Jr, Filderman PS, Jacobs SC: Ureteral injury due to blunt and penetrating trauma. Urology. 1992; 40:216-20.

3. Presti JC Jr, Carroll PR, McAninch JW: Ureteral and renal pelvic injuries from external trauma: diagnosis and management. J Trauma. 1989; 29: 370-4.

4. Palmer LS, Rosenbaum RR, Gershbaum MD, Kreutzer ER: Penetrating ureteral trauma at an urban trauma center: 10-year experience. Urology. 1999; 54: 34-6.

5. Holden S, Hicks CC, O'Brien DP, Stone HH, Walker JA, Walton KN: Gunshot wounds of the ureter: a 15-year review of 63 consecutive cases. J Urol. 1976; 116: 562-4.

6. Medina D, Lavery R, Ross SE, Livingston DH: Ureteral trauma: preoperative studies neither predict injury nor prevent missed injuries. J Am Coll Surg. 1998; 186: 641-4.

7. Velmahos GC, Degiannis E, Wells M, Souter I: Penetrating ureteral injuries: the impact of associated injuries on management. Am Surg. 1996; 62: 461-8.

8. Ghali AM, El Malik EM, Ibrahim AI, Ismail G, Rashid M: Ureteric injuries: diagnosis, management, and outcome. J Trauma. 1999; 46: 150-8.

9. Guerriero WG: Ureteral injury. Urol Clin North Am. 1989; 16: 237-48.

10. McAninch JW, Santucci RA. Genitourinary trauma. In: Walsh PC, Retik AB, Vaughan, EDJ (Eds.), Campbell's Urology. Eighth edition. New York, Saunders. 2002; 4: 3707-3744.

11. Champion HR, Sacco WJ, Copes WS, Gann DS, Gennarelli TA, Flanagan ME: A revision of the Trauma Score. J Trauma. 1989; 29: 623-9.
12. Borlase BC, Moore EE, Moore FA: The abdominal trauma index - a critical reassessment and validation. J Trauma. 1990; 30: 1340-4.

13. Baker SP, O'Neill B, Haddon W Jr, Long WB: The injury severity score: a method for describing patients with multiple injuries and evaluating emergency care. J Trauma. 1974; 14: 187-96.

14. Boyd CR, Tolson MA, Copes WS: Evaluating trauma care: the TRISS method. Trauma Score and the Injury Severity Score. J Trauma. 1987; 27: 370-8.

15. Moore EE, Cogbill TH, Jurkovich GJ, McAninch JW, Champion HR, Gennarelli TA, et al.: Organ injury scaling. III: Chest wall, abdominal vascular, ureter, bladder, and urethra. J Trauma. 1992; 33: 337-9.

16. Kotkin L, Brock JW 3rd: Isolated ureteral injury caused by blunt trauma. Urology. 1996; 47: 111-3.

17. Perez-Brayfield MR, Keane TE, Krishnan A, Lafontaine P, Feliciano DV, Clarke HS: Gunshot wounds to the ureter: a 40-year experience at Grady Memorial Hospital. J Urol. 2001; 166: 119-21.

18. Azimuddin K, Milanesa D, Ivatury R, Porter J, Ehrenpreis M, Allman DB: Penetrating ureteric injuries. Injury. 1998; 29: 363-7.

19. Mendez R, McGinty DM: The management of delayed recognized ureteral injuries. J Urol. 1978; 119: 192-3.

20. Cormio L, Battaglia M, Traficante A, Selvaggi FP: Endourological treatment of ureteric injuries. Br J Urol. 1993; 72: 165-8.

21. Best CD, Petrone P, Buscarini M, Demiray S, Kuncir E, Kimbrell B, et al.: Traumatic ureteral injuries: a single institution experience validating the American Association for the Surgery of Trauma-Organ Injury Scale grading scale. J Urol. 2005; 173: 1202-5.

\section{Correspondence address:}

Dr. Gustavo Pereira Fraga

Rua Cel. Silva Teles, 211 / 3

Campinas, SP, 13024-000, Brazil

Fax: + 55 193788-7481

E-mail: fragagp@uol.com.br 


\section{EDITORIAL COMMENT}

The authors retrospectively review their eightyear experience with the management of penetrating ureteral trauma. Their observations are well-supported by several earlier case series, including our own (1). Ureteral injuries are rare and are often present without hematuria or hypotension. Thus, a high index of suspicion is warranted in the evaluation of any patient with retroperitoneal trauma, particularly from gunshot wounds as the blast effect can have consequences up to $2 \mathrm{~cm}$ away from the path of the bullet. My practice is to stent all contusions due to blast effect as this often progresses to necrosis in the subsequent days and the defect will heal well over a stent.

As the authors suggest, the best radiographic techniques for the identification of ureteral trauma include retrograde pyelogram or computerized tomography (CT) with delayed pyelogram-phase views. When laparotomy is warranted for other reasons it is ill-advised to delay surgery in order to perform these time-consuming tests. As such, we are often presented with the dilemma of evaluating retroperitoneal hematoma without the aid of highquality imaging. An on-table one-shot intravenous pyelogram can be performed in the operating theater but is insensitive for the diagnosis of ureteral injury. Thus, when the path of the bullet aligns with the known path of the ureter, retroperitoneal exploration is indicated in order to rule out a ureteral injury. As the authors describe, visual inspection is key and can be augmented by injection of methylene blue. Another technique is to make a cystotomy and pass catheters up the ureters.
There are rare cases when penetrating trauma occurs along the known course of the ureter but opening of the retroperitoneum is felt to be contraindicated (i.e. due to fear of releasing a contained hematoma after pelvic fracture or iliac vein injury) or when the patient's hemodynamic status does not allow a thorough exploration of the ureter. In these cases every effort should be made to perform retrograde pyelograms with possible stent placement in the operating theater at the time of laparotomy or to obtain imaging with CT scan or retrograde pyelograms within the first postoperative day. When transporting the unstable patient out of the Intensive Care Unit on postoperative day \#1 is not possible, I have performed retrograde pyelograms at the bedside using a flexible cystoscope and portable kidneys, ureters and bladder (KUB) $\mathrm{x}$-ray. With due vigilance we can strive to minimize the delayed presentation of ureteral injury. Fortunately, the authors were able to endoscopically manage both patients who presented with delayed injury. In the case of delayed presentation of a complete transection that can not be managed with ureteral stenting, one should consider a percutaneous nephrostomy tube with delayed reconstruction rather than pursue repeat laparotomy in the polytrauma patient.

\section{REFERENCE}

1. Elliott SP, McAninch JW: Ureteral injuries from external violence: the 25-year experience at San Francisco General Hospital. J Urol. 2003; 170:1213-6.

Dr. Sean P. Elliott Assistant Professor of Urology University of Minnesota Minneapolis, Minnesota, USA E-mail: selliott@umn.edu 


\section{EDITORIAL COMMENT}

The authors of this paper have described their experience in identifying and managing acute ureteral injuries secondary to external trauma. This is a retrospective review of their institutions experience with penetrating ureteral injuries, thoroughly evaluating associated factors and trends. Although not an original addition, I think it adds reinforcing value to recent literature. Their findings as far as incidence of hematuria, method of diagnosis, and management options are comparable to those documented by other series. (1-3). I do have a comment in regards to their intraoperative success in diagnosing the ureteral injury. All 20 patients in this series went directly to exploratory laparotomy, despite $75 \%$ being hemodynamically stable. Perhaps if these patients had preoperative imaging, particularly with a contrast-enhanced CT scan with delayed images, there would have been no missed injuries. Although their overall complication rate seems high, this can be due to the severity of injury that their population obtained. The percentage of uro- logic specific injuries is consistent with other series. Overall, I believe this to be a very well done study, highlighting the importance of having a high index of suspicion for ureteral injuries and assessing the need for management based on grade of injury and timing of diagnosis. This data will contribute to the validity of the AAST-OIS grading scale for ureteral injury.

\section{REFERENCES}

1. Presti JC Jr, Carroll PR, McAninch JW: Ureteral and renal pelvic injuries from external trauma: diagnosis and management. J Trauma. 1989; 29:370-4.

2. Best CD, Petrone P, Buscarini M, Demiray S, Kuncir E, Kimbrell B, et al: Traumatic ureteral injuries: a single institution experience validating the American Association for the Surgery of Trauma-Organ Injury Scale. J Urol. 2005; 173: 1202-5.

3. Palmer LS, Rosenbaum RR, Gershbaum MD, Kreutzer ER: Penetrating ureteral trauma at an urban trauma center: 10-year experience. Urology. 1999; 54:34-6.

Dr. Charles D. Best Chief, Department of Urology Los Angeles County and Univ. Southern California LAC/USC County Medical Center Los Angeles, California, USA E-mail: cbest@usc.edu 